\title{
Proceedings of the Institution of Civil Engineers
}

\section{ice publishing}

\section{Corrigendum}

(1971) Obituary of Dr John Holmes Jellett. Proceedings of the Institution of Civil Engineers 49(4): 588-589, https://doi. org/10.1680/iicep.1971.32576
The date of Dr John Holmes Jellett's death is incorrect in the text. The first paragraph of this obituary should begin:

'Dr John Holmes Jellett, OBE, DSc, MA

a Past-President of the Institution, died on 17 June, 1971.' 